\title{
Adaptive Energy Budgeting for Atomic Operations in Intermittently-Powered Systems
}

\author{
Jie Zhan \\ University of Southampton \\ Southampton, United Kingdom \\ j.zhan@soton.ac.uk
}

\author{
Alex S. Weddell \\ University of Southampton \\ Southampton, United Kingdom \\ asw@ecs.soton.ac.uk
}

\author{
Geoff V. Merrett \\ University of Southampton \\ Southampton, United Kingdom \\ gvm@ecs.soton.ac.uk
}

\begin{abstract}
Intermittently-powered energy-harvesting systems offer the potential to form a long-lasting and environmentally friendly Internet of Things. Atomic operations in such systems refer to those that should be executed without interruption, e.g. wireless transmission; if interrupted by power loss, they must restart rather than resume from the interrupted point. State-of-the-art approaches handle atomic operations by either taking a checkpoint before the operation, or ensuring sufficient energy is buffered through design-time profiling of the required energy. Such approaches become inefficient or fail if energy consumption or storage changes, e.g. due to aging or environmental factors. This paper proposes a method for online profiling of energy budgets for atomic operations, enabling intermittent systems to adapt to changes in environment or system conditions. We show that an existing approach fails if capacitance drops by $66 \%$, whereas our approach successfully adapts to changes and ensures atomicity as long as the required energy can be stored.
\end{abstract}

\section{CCS CONCEPTS}

\section{- Computer systems organization $\rightarrow$ Embedded systems.}

\section{KEYWORDS}

energy harvesting, intermittent systems, online profiling

\section{ACM Reference Format:}

Jie Zhan, Alex S. Weddell, and Geoff V. Merrett. 2020. Adaptive Energy Budgeting for Atomic Operations in Intermittently-Powered Systems. In The 8th International Workshop on Energy Harvesting and Energy-Neutral Sensing Systems (ENSsys '20), November 16-19, 2020, Virtual Event, Japan. ACM, New York, NY, USA, 2 pages. https://doi.org/10.1145/nnnnnnn.nnnnnnn

\section{INTRODUCTION}

Battery-free devices are a promising option for constructing an environmentally friendly, maintenance-free, and sustainable Internet of Things. Energy harvesters enable low-power electronic systems to operate from environmental energy sources rather than battery power. A class of such systems containing virtually no energy storage, e.g. only enough to sustain operation for milliseconds, are known as intermittent systems, where program execution is performed only when environmental energy is intermittently available, and the system is off for the remainder of the time. Much of the recent research in intermittent systems has focused on addressing memory consistency and efficiency of computational workloads [3]

However, in practical applications, embedded systems typically need to utilize peripheral resources, such as sensors, computational

ENSsys '20, November 16-19, 2020, Virtual Event, Japan 2020. ACM ISBN 978-1-4503-8129-1/20/11...\$15.00

https://doi.org/10.1145/nnnnnnn.nnnnnnn accelerators, and radios. Operations on peripherals usually require atomicity, meaning that the operation should be completed in one continuous period, and should restart rather than resume if interrupted when power fails. The atomicity requirement may be due to unobtainable peripheral internal memory, time constraints, or a series of memory writes. For instance, a radio can have its own internal state, send data that might expire, and listen to other nodes in a scheduled period.

State-of-the-art techniques handle atomic operations by checkpointing computing state before the atomic operations, or offline profiling of energy budgets for each operation. The checkpointing method makes a checkpoint before an atomic operation and disables checkpoints during its execution [2]. When power fails, it restores the checkpoint to restart the operation. Re-executing the operation may waste time and energy, making it inefficient. Offline profiling measures the energy consumption of each atomic operation at design time, and the system waits for the stored energy to exceed the profiled threshold before beginning the atomic operation [1]. This may leave the program stuck if the energy consumption or storage changes at run time due to aging or environmental factors (e.g. temperature).

In this paper, we propose a methodology for online profiling of the required energy for atomic operations in intermittent systems, enabling them to adapt to changes in the energy consumption or storage.

\section{SYSTEM DESIGN}

The proposed method enables intermittent systems to profile the energy consumption of code segments at run time, so as to adapt execution to changes in energy capacity or consumption.

The system circuit is shown in Figure 1. It utilizes an N-channel FET to short-circuit the energy harvesting supply during calibration, with a pull-down resistor to keep the gate low when MCU is not powered. The supply is a photovoltaic (PV) panel, which is decoupled by a diode to prevent current backflow. The MSP430FR5994 microcontroller (MCU) uses its internal comparator and ADC to monitor and measure a divided supply voltage $\frac{1}{3} V_{c c}$.

The system automatically takes a checkpoint when $V_{c c}$ falls below a backup threshold during non-atomic computation, while performing the proposed approach for atomic segments. The handling routine of atomic segments is shown in Figure 2. At the entry to an atomic segment, the routine switches to calibration if the atomic segment fails in the last execution or is not calibrated. Execution failure is checked by setting and resetting a nonvolatile variable when the atomic segment begins and finishes.

Entering the calibration process, the system stays in low-power mode until $V_{c c}$ reaches a calibration threshold $V_{\text {calibrate }}$, which 


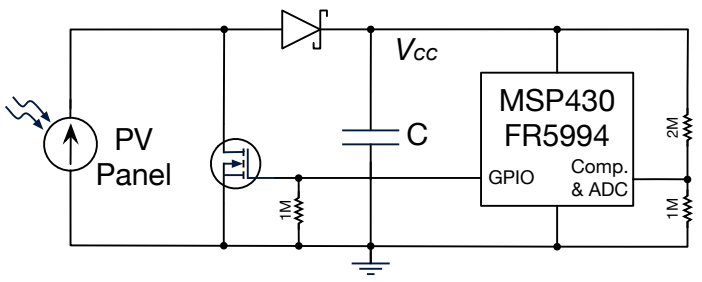

Figure 1: Experimental schematic.

can be determined by the maximum operating threshold of the MCU and the energy harvester. Next, the MCU takes a sample of $V_{c c}\left(V_{1}\right)$, disables the automatic checkpoints, turns on the FET to short-circuit the supply, and start the atomic segment. Afterwards, the system enables checkpoints, reconnects the supply, and samples $V_{c c}$ again $\left(V_{2}\right)$. The calibrated threshold $V_{t h}$ is calculated and updated as:

$$
V_{t h}=\operatorname{Roundup}\left(V_{1}-V_{2}+V_{\text {min }}\right)
$$

where Roundup() takes the lowest available threshold beyond its input value and allows a safe margin, and $V_{\text {min }}$ is the minimum operating voltage of the platform, which is $1.8 \mathrm{~V}$ for MSP430FR5994. In the following execution of the atomic segment, the system waits for its calibrated $V_{t h}$ until a failure happens due to changes in energy storage or consumption.

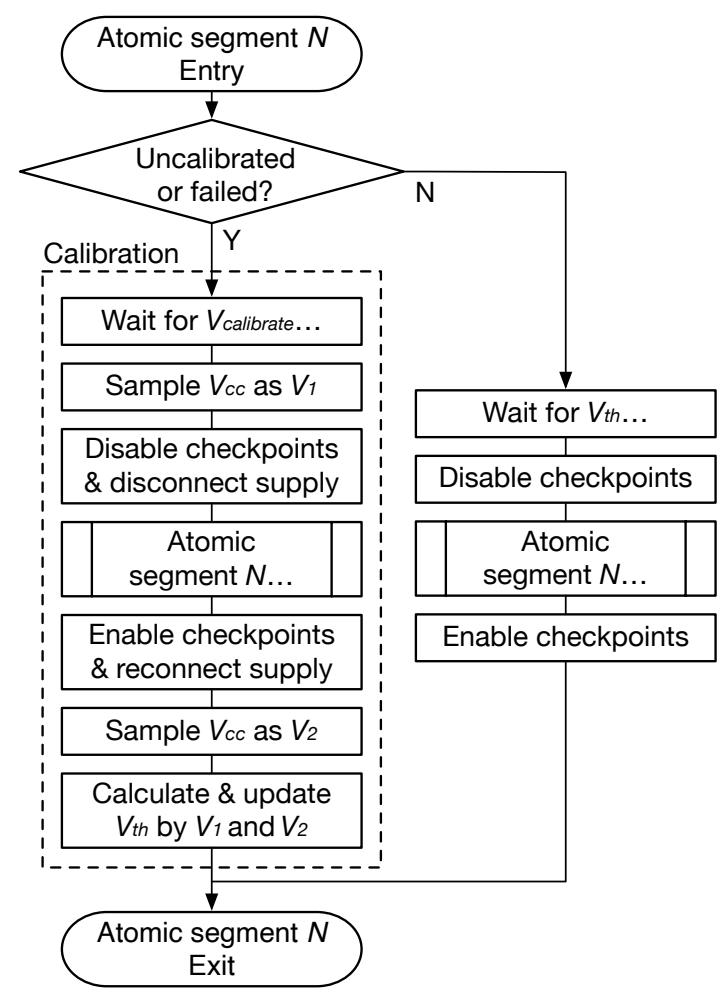

Figure 2: Handling routine of atomic segments.

\section{EVALUATION RESULTS}

Our benchmark is a function of 128-bit AES encryption performed with the MCU's built-in AES accelerator, processing $2 \mathrm{kB}$ data in $26.2 \mathrm{~ms}$. The voltage drop of this function was $0.25 \mathrm{~V}$ with $30 \mu \mathrm{F}$ capacitance, so the offline profiled threshold is set to $2.1 \mathrm{~V}$, allowing a $0.05 \mathrm{~V}$ margin. The platform was powered by a PV panel with a constant indoor light source that provided $140 \mu \mathrm{A}$ at $2 \mathrm{~V}$. We set the calibration threshold $V_{\text {calibrate }}$ to $3.0 \mathrm{~V}$.

We initially provided $30 \mu \mathrm{F}$ energy capacity and decreased that by $10 \mu \mathrm{F}$ each time to test adaptivity. The result is shown in Figure 3. Initially, our proposed approach calibrated the same threshold as the offline approach does, rendering the same performance. With $20 \mu \mathrm{F}$ capacitance, the offline approach can still work given that the PV panel gives power during execution to keep $V_{c c}$ above $V_{\text {min }}$, but that can fail in the worst case where the supply current instantaneously drops to zero just after the atomic segment starts.

With $10 \mu \mathrm{F}$ capacitance, the offline approach kept failing and rebooting, and could never complete the atomic segment. However, the proposed approach re-calibrated the threshold to $2.45 \mathrm{~V}$ after a failure, hence guaranteeing atomicity and maintaining forward progress. This adapts the system to a smaller energy capacity.

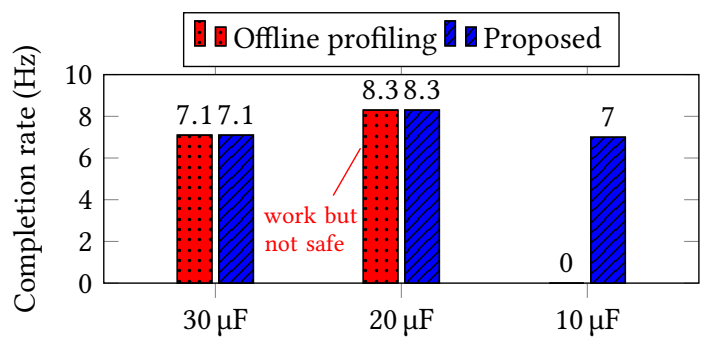

Figure 3: Completion rate of the proposed approach and the offline profiling approach.

\section{CONCLUSIONS}

This paper has proposed an approach to perform online profiling of the energy required to execute atomic tasks, allowing the system to adapt its operation to changes in energy capacity and consumption at runtime. Our current work is exploring the policies for routine and predictive recalibration of the required energy.

\section{ACKNOWLEDGMENTS}

This research was supported in part by the UK Engineering and Physical Sciences Research Council (EPSRC) under EP/P010164/1. All available data for this study have been stated in this paper.

\section{REFERENCES}

[1] A. Gomez, L. Sigrist, M. Magno, L. Benini, and L. Thiele. 2016. Dynamic energy burst scaling for transiently powered systems. In 2016 Design, Automation Test in Europe Conference Exhibition (DATE). 349-354.

[2] Kiwan Maeng and Brandon Lucia. 2019. Supporting Peripherals in Intermittent Systems with Just-in-Time Checkpoints. In Proceedings of the 40th ACM SIGPLAN Conference on Programming Language Design and Implementation (Phoenix, AZ, USA) (PLDI 2019). 1101-1116.

[3] Sivert T. Sliper, Oktay Cetinkaya, Alex S. Weddell, Bashir Al-Hashimi, and Geoff V. Merrett. 2020. Energy-driven computing. Philosophical Transactions of the Royal Society A 378, 2164 (2020). 